\title{
A double-blind comparison of topical therapy of chlamydial ocular infection (TRIC infection) with rifampicin or chlortetracycline
}

\author{
S. DAROUGAR, N. VISWALINGAM, H. EL-SHEIKH, P. A. HUNTER, AND \\ P. YEARSLEY \\ From the External Eye Diseases Clinic, Moorfield's Eye Hospital, City Road, London ECIV 2PD, \\ and the Subdepartment of Virology, Institute of Ophthalmology, Judd Street, London WCIH $9 Q S$
}

SUMMARY A double-blind stratified trial was carried out on 85 patients to assess the efficacy of topical therapy with $1 \%$ chlortetracycline eye ointment in comparison with $1 \%$ rifampicin eye ointment in the treatment of chlamydial ocular infection of sexually transmitted origin (paratrachoma). Patients included were selected on the basis of positive culture for Chlamydia trachomatis. A 6-week course of treatment with chlortetracycline or rifampicin 3 times daily gave a clinical cure rate of $80 \%$ and $75 \%$ and a microbiological cure rate of $93 \%$ and $86 \%$ respectively. In patients who were not cured the intensity of inflammatory responses was considerably reduced.

Chlamydial ocular infection of sexually transmitted origin (paratrachoma or TRIC ocular infection) is becoming commoner in the urban populations of both developed and developing countries. This is because of a substantial increase in the incidence of chlamydial genital infections, that is, chlamydial urethritis and chlamydial cervicitis.

In a previous paper $^{1}$ it was shown that topical therapy of paratrachoma with rifampicin eye ointment produced a clinical and microbiological cure rate of $90 \%$. In this paper we present the result of a double-blind stratified trial to assess the efficacy of topical therapy of paratrachoma with rifampicin eye ointment compared with chlortetracycline eye ointment.

\section{Subjects and methods}

Selection of patients. Patients attending the External Eye Diseases Clinic, Moorfield's Eye Hospital, with acute follicular conjunctivitis and a positive culture for Chlmaydia trachomatis were selected. Excluded from this study were patients who had symptoms of concurrent genital infection, or who had been treated with antibiotics known to be effective

Correspondence to Professor S. Darougar, Subdepartment of Virology, Institute of Ophthalmology, Judd Street, London WC1H 9QS. against chlamydiae, ${ }^{2}$ or who were unable to attend for 12 weeks after the initial visit.

Clinical examination. Patients were examined with a Haag-Streit slit-lamp. The symptoms and signs in the conjunctiva of the upper tarsus, upper fornix, and lower lid and cornea were graded on a 0 to 3 scale as described previously. ${ }^{1}$ The severity of disease was calculated from an extension of the scoring system used for trachoma. ${ }^{3}$

Laboratory investigations. The methods of collection of conjunctival swabbings, transport to the laboratory, culture in irradiated McCoy cells, and identification of isolates have been described elsewhere. ${ }^{4} 5$

Random allocation. Patients included in the study were allocated to treatments by the Chief Pharmacist, Moorfield's Eye Hospital, according to a randomisation schedule stratified for age, sex, intensity of conjunctival inflammatory responses, and corneal involvement.

Medication. Each patient was treated with either $1 \%$ chlortetracycline eye ointment or $1 \%$ rifampicin eye ointment 3 times daily for 6 weeks. The antibiotics were supplied by Le Petit Pharmaceuticals Ltd in identical containers each labelled with a code number. Patients were requested to avoid using the eye ointment on the day they attended the clinic, until after they were examined. This was to prevent any biased observation which could occur 
Table 1 Numbers of patients cured after treatment with chlortetracycline or rifampicin eye ointment

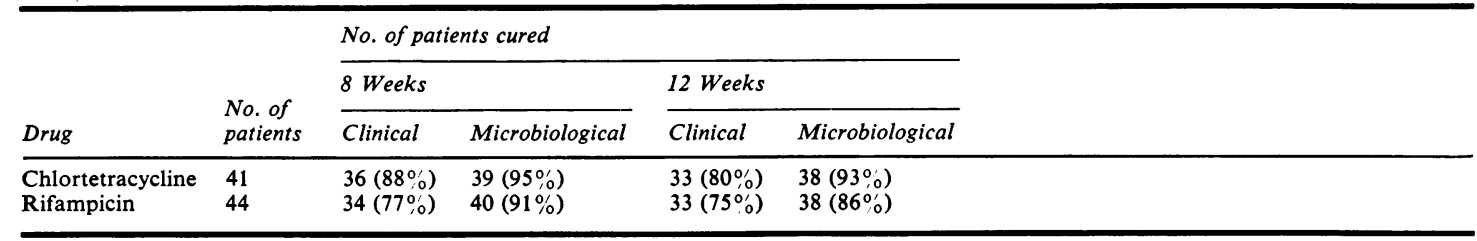

because of the red colour of rifampicin eye ointment.

Follow-up investigations. After the initial clinical and laboratory investigations, treatment was started and follow-up investigations were carried out at weekly intervals for 4 weeks, then at 6,8 , and 12 weeks. After the last visit all patients were referred to the Diagnostic Clinic, Moorfield's Eye Hospital, for further investigation and treatment of chlamydial genital infection.

Statistical analysis. Chi-squared significance tests were used for comparison of results.

\section{Results}

Eighty-five patients suffering from inclusion conjunctivitis or TRIC punctate keratoconjunctivitis were included in this study. The numbers of males and females were 53 to 32 . Their ages ranged between 15 and 48 years, the majority being 20 to 30 years old.

The clinical and microbiological findings in 41 patients treated with chlortetracycline and 44 patients treated with rifampicin are shown in Table 1 and Figs. 1 and 2.

The average clinical scores before treatment in the groups treated with chlortetracycline or rifampicin were 34 and 36 respectively. After 1 week of treatment there was a marked decrease in the clinical scores in both groups of patients (Fig. 1). The average clinical scores in these patients immediately after completion of treatment at 6 weeks was 8 for chlortetracycline and 9 for rifampicin (Fig. 1). Clinical examination at 12 weeks showed complete cure in 33 out of $41(80 \%)$ of patients treated with chlortetracycline and 33 out of $44(75 \%)$ of patients treated with rifampicin (Table 1). The beneficial effects of therapy with the 2 antibiotics were statistically significant $(p<0.001)$, but there was no difference in the efficacy of the 2 drugs $(p>0 \cdot 2)$. There was a marked improvement in the clinical condition of the patients who were not cured by chlortetracycline or rifampicin therapy. At the last examination of the 8 patients not cured with chlortetracycline 2 had moderate disease and 6 had mild disease. Microbiologically 3 of these 8 patients were positive. Of the patients who were not cured by rifampicin therapy 2 had severe disease, 2 had moderate disease, and 7 had mild disease at the last clinical examination. Microbiologically 6 of these 11 patients were positive.

There was a considerable drop in the numbers of isolation-positive patients at 1 week; only $4(10 \%)$ out of 41 patients treated with chlortetracycline and $1(2 \%)$ out of 44 treated with rifampicin were

INTENSITY OF CLINICAL SIGNS AND SYMPTOMS

Fig. 1 Changes in the average score of intensity of signs and symptoms after treatment with chlortetracycline (O) or rifampicin ( $\square$ ).

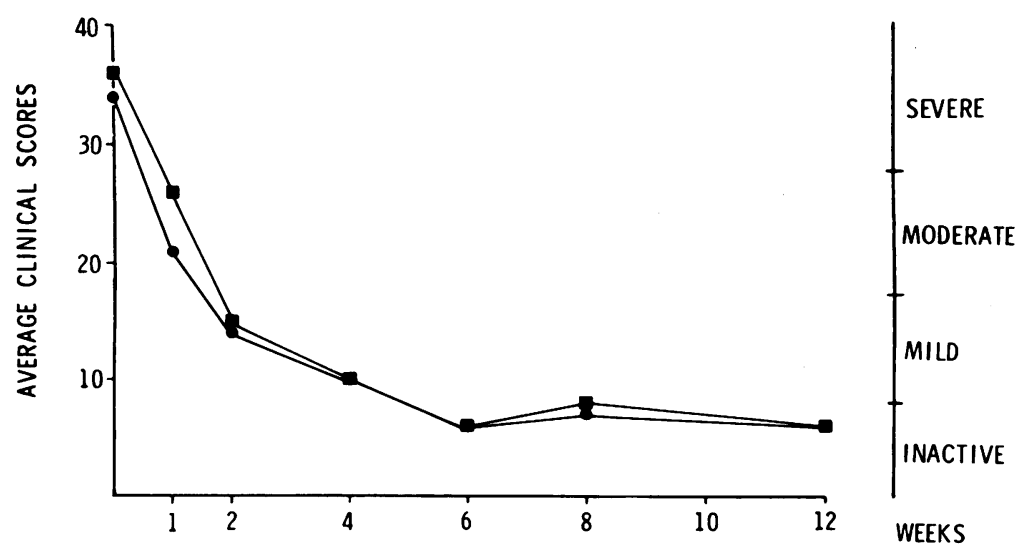


PERCENTAGE OF POSITIVE CELL CULTURES FOR C.trachomatis

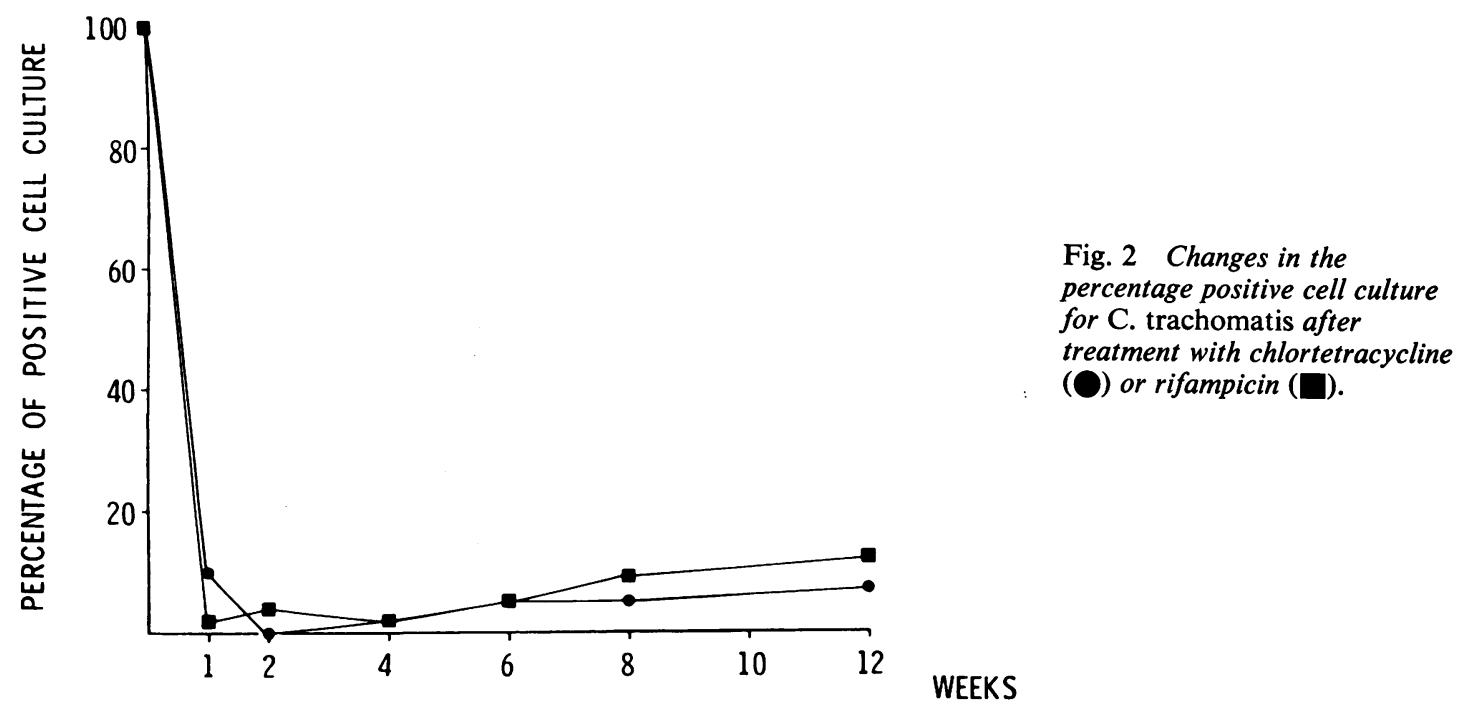

positive (Fig. 2). At 6 weeks follow-up, immediately after completion of treatment, 1 patient treated with chlortetracycline was positive, and 2 patients treated with rifampicin were positive. At 12 weeks follow-up the numbers of patients positive for $C$. trachomatis were $3(7 \%)$ and $6(14 \%)$ for chlortetracycline and rifampicin respectively (Fig. 2). All these positive patients showed active clinical signs.

\section{Discussion}

In this double-blind treatment trial stratified randomisation for grades and intensity of inflammatory responses, age, and sex produced a well balanced distribution of patients in groups allocated to treatment with chlortetracycline or rifampicin.

In the present study the whole conjunctiva including the upper tarsal area was examined for assessing the presence and intensity of inflammatory changes. This was done because the results of clinical and therapeutic studies of trachoma and paratrachoma ${ }^{6}$ ? have shown: $(a)$ in the whole conjunctiva the intensity of inflammatory responses is higher than in the upper tarsal conjunctiva; $(b)$ after treatment the cure rate for the whole conjunctiva is markedly lower than the rate for the upper tarsal conjunctiva; and $(c)$ in some patients with cured upper tarsal conjunctiva an active but mild to moderate disease could be found in the upper and lower conjunctival fornices. It was found that this residual disease can cause recurrence in treated patients.

In this study the follow-up investigations were carried out for up to 6 weeks after completion of treatment. This was done because in a previous study of topical therapy of paratrachoma with rifampicin eye ointment ${ }^{1}$ we found that clinical and microbiological recurrences generally occur within 6 weeks after completion of treatment.

The results of this investigation show that topical therapy of paratrachoma with chlortetracycline or rifampicin eye ointment, 3 times daily for 6 weeks, is highly effective (Table 1). Rifampicin produced clinical and microbiological cure rates of $75 \%$ and $86 \%$ respectively. These rates are similar to those obtained in a previous study. ${ }^{1}$ Chlortetracycline also produced very high clinical and microbiological cure rates of $80 \%$ and $93 \%$ respectively. Although this regimen of therapy has been recommended as a standard method of treatment of paratrachoma, we have not found any well-documented reports to support its efficacy.

In the present trial between 20 and $25 \%$ of patients were not cured clinically after treatment with either chlortetracycline or rifampicin (Table 1). Although an initial clinical improvement was observed in all these patients, moderate to severe recurrences were observed in only 2 patients $(5 \%)$ treated with chlortetracycline and 4 patients $(9 \%)$ treated with rifampicin. While it is possible that drug resistance may have developed in these cases, we have made no attempt to investigate this. In a previous study ${ }^{1}$ we found that resistance to rifampicin had not developed in $C$. trachomatis isolates obtained from patients who were not cured with this 
drug. We suggest therefore that inadequate application of eye ointment may be responsible for these failures, although there was no evidence of default in the frequency of application of ointment.

The authors are grateful to colleagues at Moorfield's Eye Hospital for referring their patients and to Mr R. Watkins, Chief Pharmacist, Moorfield's Eye Hospital, for organising the random allocation of patients.

This work was financially supported by the Locally Organised Clinical Research Scheme, Moorfield's Eye Hospital, and by a grant from the Department of Health and Social Security and an anonymous donor.

\section{References}

1 Darougar S, Viswalingam N, Treharne JD, Kinnison JR, Jones BR. Treatment of TRIC infection of the eye with rifampicin or chloramphenicol. Br J Ophthalmol 1977; 61 : 255-9.
2 Treharne JD, Day Y, Yeo CK, Jones BR, Squires S. Susceptibility of chlamydiae to chemotherapeutic agents. In: Hobson D, Holmes KK, eds. Nongonococcal Urethritis and Related Infections. Washington: American Society of Microbiology, 1977: 214-22.

3 Dawson CR, Jones BR, Darougar S. Blinding and nonblinding trachoma: Assessment of intensity of upper tarsal inflammatory disease and disabling lesions. Bull WHO 1975; 52: 279-82.

4 Darougar S, Jones BR. Conjunctival swabbing for the isolation of TRIC agents (Chlamydia). Br J Ophthalmol 1971; 55: 585-90.

5 Darougar S, Kinnison JR, Jones BR. Simplified irradiated McCoy cell culture for isolation of Chlamydia. In: Nichol R, ed. Trachoma and Related Disorders Caused by Chlamydial Agents. Excerpta Medica International Congress Series 1970; 223: 64-70.

6 Darougar S, Jones BR, Viswalingam N, et al. Topical therapy of hyperendemic trachoma with rifampicin, oxytetracycline, or spiramycin eye ointments. Br J Ophthalmol 1980; 64: 37-42.

7 Editorial: Chlamydial infections of the eye. Lancet 1977; ii: $857-8$. 